\title{
Group graded Morita equivalences for wreath products
}

\author{
Virgilius-Aurelian Minuţă
}

\begin{abstract}
Starting with group graded Morita equivalences, we obtain Morita equivalences for tensor products and wreath products.

Mathematics Subject Classification (2010): 16W50, 20E22, 20C05, 20C20, 16D90, $16 \mathrm{~S} 35$.

Keywords: Group graded algebras, wreath products, Morita equivalences, crossed products, centralizer subalgebra.
\end{abstract}

\section{Introduction}

In this article, we continue the study done in [2], [3] and [4], and we obtain group graded Morita equivalences for tensor products (Proposition 3.3) and wreath products (Theorem 5.3). The main motivation for such constructions in the representation theory of finite groups is given by the fact that in order to prove most reduction theorems, recent results of Britta Späth, surveyed in [5], [6] and [7], show that a new character triple can be constructed via a wreath product construction of character triples ([7, Theorem 2.21]). There is a link between character triples and group graded Morita equivalences, presented in [3], so we want to prove that a similar wreath product construction can also be made for the corresponding group graded Morita equivalences.

More precisely, in Theorem 6.7 of [3], it is proved that certain character triples relations utilized by Britta Späth: the first-order relation ([7, Definition 2.1]) and the central-order relation ([7, Definition 2.7]), are consequences of a special type of group graded Morita equivalences induced by a graded bimodule over a $G$-graded $G$-acted algebra (usually denoted by $\mathcal{C}$ as in Section 2.3), where $G$ is a finite group. More details about group graded Morita theory over $\mathcal{C}$ can be found in [4].

Another motivation comes from the fact that it is already known by $[1$, Theorem 5.1.21] that Morita equivalences can be extended to wreath products. 
This paper is organized as follows: In Section 2, we introduce the general notations and we recall from [3] the definitions of a $G$-graded $G$-acted algebra, of a $G$-graded algebra over $\mathcal{C}$, of a $G$-graded bimodule over $\mathcal{C}$ and the notion of a $G$ graded Morita equivalence over $\mathcal{C}$. In Section 3, we prove that the previously recalled algebraic constructions are compatible with tensor products and the main proposition in this section, Proposition 3.3, proves that the tensor products of some group graded Morita equivalent algebras over some group graded group acted algebras remain group graded Morita equivalent over a group graded group acted algebra. In Section 4, we prove that the previously enumerated algebra types are also compatible with wreath products. Finally, in Section 5, our main result, Theorem 5.3, proves that the wreath product between a $G$-graded bimodule over $\mathcal{C}$ and $S_{n}$ (the symmetric group of order $n$ ) is also a group graded bimodule over $\mathcal{C}^{\otimes n}$, and moreover, if this bimodule induces a $G$-graded Morita equivalence over $\mathcal{C}$, then its wreath product with $S_{n}$ will induce a group graded Morita equivalence over $\mathcal{C}^{\otimes n}$.

\section{Preliminaries}

2.1. All rings in this paper are associative with identity $1 \neq 0$ and all modules are left (unless otherwise specified) unital and finitely generated. Throughout this article $n$ will represent an arbitrary nonzero natural number, and $\mathcal{O}$ is a commutative ring.

2.2. Let $G$ be a finite group and $N$ a normal subgroup of $G$. We denote by $\bar{G}:=G / N$. Note that most results in this paper will utilize " $G$-gradings", although this is not essential: one may consider instead the gradings to be given directly by $G$. The reasoning behind this particular choice is to match our notations previously used in articles [2] and [3], given that our main application for the results of this project is the strongly $\bar{G}$-graded algebra $A=b \mathcal{O} G$, where $b$ is a $\bar{G}$-invariant block of $\mathcal{O} N$.

2.3. We recall from [3] the following definitions:

Definition 2.4. An algebra $\mathcal{C}$ is a $\bar{G}$-graded $\bar{G}$-acted algebra if

(1) $\mathcal{C}$ is $\bar{G}$-graded, and we write $\mathcal{C}=\bigoplus_{\bar{g} \in \bar{G}} \mathcal{C}_{\bar{g}}$;

(2) $\bar{G}$ acts on $\mathcal{C}$ (always on the left in this article);

(3) for all $\bar{g}, \bar{h} \in \bar{G}$ and for all $c \in \mathcal{C}_{\bar{h}}$ we have ${ }^{\bar{g}} c \in \mathcal{C}_{\bar{g}}{ }_{h}$.

Definition 2.5. Let $\mathcal{C}$ be a $\bar{G}$-graded $\bar{G}$-acted algebra. We say the $A$ is a $\bar{G}$-graded algebra over $\mathcal{C}$ if there is a $\bar{G}$-graded $\bar{G}$-acted algebra homomorphism

$$
\zeta: \mathcal{C} \rightarrow C_{A}(B)
$$

where $B:=A_{1}$ and $C_{A}(B)$ is the centralizer of $B$ in $A$, i.e. for any $\bar{h} \in \bar{G}$ and $c \in \mathcal{C}_{\bar{h}}$, we have $\zeta(c) \in C_{A}(B)_{\bar{h}}$, and for every $\bar{g} \in \bar{G}, \zeta\left({ }^{\bar{g}} c\right)={ }^{\bar{g}} \zeta(c)$.

Definition 2.6. Let $A$ and $A^{\prime}$ be two $\bar{G}$-graded crossed products over a $\bar{G}$-graded $\bar{G}$-acted algebra $\mathcal{C}$, with structure maps $\zeta$ and $\zeta^{\prime}$, respectively.

a) We say that $\tilde{M}$ is a $\bar{G}$-graded $\left(A, A^{\prime}\right)$-bimodule over $\mathcal{C}$ if:

(1) $\tilde{M}$ is an $\left(A, A^{\prime}\right)$-bimodule; 
(2) $\tilde{M}$ has a decomposition $\tilde{M}=\bigoplus_{\bar{g} \in \bar{G}} \tilde{M}_{\bar{g}}$ such that $A_{\bar{g}} \tilde{M}_{\bar{x}} A_{\bar{h}}^{\prime} \subseteq \tilde{M}_{\bar{g} \bar{x} \bar{h}}$, for all $\bar{g}, \bar{x}, \bar{h} \in \bar{G}$

(3) $\tilde{m}_{\bar{g}} c=\bar{g}_{c} \tilde{m}_{\bar{g}}$, for all $c \in \mathcal{C}, \tilde{m}_{\bar{g}} \in \tilde{M}_{\bar{g}}, \bar{g} \in \bar{G}$, where $c \tilde{m}=\zeta(c) \tilde{m}$ and $\tilde{m} c=\tilde{m} \zeta^{\prime}(c)$, for all $c \in \mathcal{C}, \tilde{m} \in \tilde{M}$.

b) $\bar{G}$-graded $\left(A, A^{\prime}\right)$-bimodules over $\mathcal{C}$ form a category, where the morphisms between $\bar{G}$-graded $\left(A, A^{\prime}\right)$-bimodules over $\mathcal{C}$ are just homomorphisms between $\bar{G}$ graded $\left(A, A^{\prime}\right)$-bimodules.

Definition 2.7. Let $A$ and $A^{\prime}$ be two $\bar{G}$-graded crossed products over a $\bar{G}$-graded $\bar{G}$-acted algebra $\mathcal{C}$, and let $\tilde{M}$ be a $\bar{G}$-graded $\left(A, A^{\prime}\right)$-bimodule over $\mathcal{C}$. Clearly, the $A$-dual $\tilde{M}^{*}=\operatorname{Hom}_{A}(\tilde{M}, A)$ of $\tilde{M}$ is a $\bar{G}$-graded $\left(A^{\prime}, A\right)$-bimodule over $\mathcal{C}$. We say that $\tilde{M}$ induces a $\bar{G}$-graded Morita equivalence over $\mathcal{C}$ between $A$ and $A^{\prime}$, if $\tilde{M} \otimes_{A^{\prime}} \tilde{M}^{*} \simeq A$ as $\bar{G}$-graded $(A, A)$-bimodules over $\mathcal{C}$ and if $\tilde{M}^{*} \otimes_{A} \tilde{M} \simeq A^{\prime}$ as $\bar{G}$-graded $\left(A^{\prime}, A^{\prime}\right)$ bimodules over $\mathcal{C}$.

\section{Tensor products}

3.1. Consider $G_{i}$ to be a finite group, $N_{i}$ to be a normal subgroup of $G_{i}$ and denote by $\bar{G}_{i}=G_{i} / N_{i}$, for all $i \in\{1, \ldots, n\}$. We denote by

$$
\overline{\mathbf{G}}:=\prod_{i=1}^{n} \bar{G}_{i} .
$$

Lemma 3.2. Let $A_{i}$ be $\bar{G}_{i}$-graded algebras and $\mathcal{C}_{i}$ be $\bar{G}_{i}$-graded $\bar{G}_{i}$-acted algebras, for all $i \in\{1, \ldots, n\}$. The following affirmations hold:

(1) The tensor product $\mathbf{A}:=A_{1} \otimes \ldots \otimes A_{n}$ is a $\overline{\mathbf{G}}$-graded algebra;

(2) If $A_{i}$ are $\bar{G}_{i}$-graded crossed products, for all $i \in\{1, \ldots, n\}$, then $\mathbf{A}$ is a $\overline{\mathbf{G}}$-graded crossed product;

(3) The tensor product $\mathcal{C}:=\mathcal{C}_{1} \otimes \ldots \otimes \mathcal{C}_{n}$ is a $\overline{\mathbf{G}}$-graded $\overline{\mathbf{G}}$-acted algebra;

(4) If $A_{i}$ are $\bar{G}_{i}$-graded algebras over $\mathcal{C}_{i}$, for all $i \in\{1, \ldots, n\}$, then $\mathbf{A}$ is a $\overline{\mathbf{G}}$-graded algebra over $\mathcal{C}$.

Proof. (1) It is clear that $\mathbf{A}$ is a $\overline{\mathbf{G}}$-graded algebra, with the $\left(g_{1}, \ldots, g_{n}\right)$-component

$$
\mathbf{A}_{\left(g_{1}, \ldots, g_{n}\right)}:=A_{1, g_{1}} \otimes \ldots \otimes A_{n, g_{n}},
$$

where $A_{i, g_{i}}$ is the $g_{i}$-component of $A_{i}$, for all $g_{i} \in \bar{G}_{i}$ and for all $i$.

(2) Choose invertible homogeneous elements $u_{i, g}$ in each $A_{i, g}$, for all $g \in \bar{G}_{i}$ and for all $i$. Thus, for each $\left(g_{1}, \ldots, g_{n}\right) \in \overline{\mathbf{G}}$ the homogeneous element

$$
u_{\left(g_{1}, \ldots, g_{n}\right)}:=u_{1, g_{1}} \otimes \ldots \otimes u_{n, g_{n}} \in \mathbf{A}_{\left(g_{1}, \ldots, g_{n}\right)}
$$

is clearly invertible.

(3) The $\overline{\mathbf{G}}$-grading of $\mathcal{C}$ is a given by (1). The action of $\overline{\mathbf{G}}$ on $\mathcal{C}$ is defined by

$$
{ }^{\left(g_{1}, \ldots, g_{n}\right)}\left(a_{1} \otimes \ldots \otimes a_{n}\right):={ }^{g_{1}} a_{1} \otimes \ldots \otimes{ }^{g_{n}} a_{n},
$$


for all $\left(g_{1}, \ldots, g_{n}\right) \in \overline{\mathbf{G}}$ and $a_{1} \otimes \ldots \otimes a_{n} \in \mathcal{C}$. It is easy too see that for all $\left(g_{1}, \ldots, g_{n}\right),\left(h_{1}, \ldots, h_{n}\right) \in \overline{\mathbf{G}}$ and for all $a_{1} \otimes \ldots \otimes a_{n} \in \mathcal{C}_{\left(h_{1}, \ldots, h_{n}\right)}$ we have

$$
{ }^{\left(g_{1}, \ldots, g_{n}\right)}\left(a_{1} \otimes \ldots \otimes a_{n}\right) \in \mathcal{C}_{\left(g_{1}, \ldots, g_{n}\right)}\left(h_{1}, \ldots, h_{n}\right) .
$$

(4) By part (1), the identity component of $\mathbf{A}$ is $\mathbf{B}=B_{1} \otimes \ldots \otimes B_{n}$, where $B_{i}$ is the identity component of $A_{i}$, for all $i$.

By the assumptions, we have the $\bar{G}_{i}$-graded $\bar{G}_{i}$-acted structure homomorphisms

$$
\zeta_{i}: \mathcal{C}_{i} \rightarrow C_{A_{i}}\left(B_{i}\right)
$$

for all $i$. We define $\zeta: \mathcal{C} \rightarrow C_{\mathbf{A}}(\mathbf{B})$ by

$$
\zeta\left(a_{1} \otimes \ldots \otimes a_{n}\right)=\zeta_{1}\left(a_{1}\right) \otimes \ldots \otimes \zeta_{n}\left(a_{n}\right),
$$

for all $a_{1} \otimes \ldots \otimes a_{n} \in \mathcal{C}$. It is easy to prove that $\zeta$ verifies the conditions of Definition 2.5 .

Proposition 3.3. Assume that $\mathcal{C}_{i}$ are $\bar{G}_{i}$-graded $\bar{G}_{i}$-acted algebras and that $A_{i}$ and $A_{i}^{\prime}$ are $\bar{G}_{i}$-graded crossed products over $\mathcal{C}_{i}$, for all $i \in\{1, \ldots, n\}$. If $A_{i}$ and $A_{i}^{\prime}$ are $\bar{G}_{i^{-}}$ graded Morita equivalent over $\mathcal{C}_{i}$, and if $\tilde{M}_{i}$ is a $\bar{G}_{i}$-graded $\left(A_{i}, A_{i}^{\prime}\right)$-bimodule over $\mathcal{C}_{i}$, that induces the said equivalence, for all $i$, then:

(1) $\tilde{\mathbf{M}}:=\tilde{M}_{1} \otimes \ldots \otimes \tilde{M}_{n}$ is a $\overline{\mathbf{G}}$-graded $\left(\mathbf{A}, \mathbf{A}^{\prime}\right)$-bimodule over $\mathcal{C}$, where

$\mathbf{A}:=A_{1} \otimes \ldots \otimes A_{n}, \mathbf{A}^{\prime}:=A_{1}^{\prime} \otimes \ldots \otimes A_{n}^{\prime}$ and $\mathcal{C}:=\mathcal{C}_{1} \otimes \ldots \otimes \mathcal{C}_{n}$

(2) $\tilde{\mathbf{M}}$ induces a $\overline{\mathbf{G}}$-graded Morita equivalence over $\mathcal{C}$ between $\mathbf{A}$ and $\mathbf{A}^{\prime}$.

Proof. (1) By Lemma 3.2, $\mathbf{A}$ and $\mathbf{A}^{\prime}$ are $\overline{\mathbf{G}}$-graded crossed products over $\mathcal{C}$.

Obviously, $\tilde{\mathbf{M}}$ is a $\overline{\mathbf{G}}$-graded $\left(\mathbf{A}, \mathbf{A}^{\prime}\right)$-bimodule with the $\left(g_{1}, \ldots, g_{n}\right)$-component

$$
\tilde{\mathbf{M}}_{\left(g_{1}, \ldots, g_{n}\right)}:=\tilde{M}_{1, g_{1}} \otimes \ldots \otimes \tilde{M}_{n, g_{n}},
$$

where $\tilde{M}_{i, g_{i}}$ is the $g_{i}$-component of $\tilde{M}_{i}$, for all $g_{i} \in \bar{G}_{i}$ and for all $i$. It is also clear that

$$
\left(\tilde{m}_{1, g_{1}} \otimes \ldots \otimes \tilde{m}_{n, g_{n}}\right)\left(c_{1} \otimes \ldots \otimes c_{n}\right)={ }^{g}\left(c_{1} \otimes \ldots \otimes c_{n}\right)\left(\tilde{m}_{1, g_{1}} \otimes \ldots \otimes \tilde{m}_{n, g_{n}}\right),
$$

for all $\tilde{m}_{1, g_{1}} \otimes \ldots \otimes \tilde{m}_{n, g_{n}} \in \tilde{\mathbf{M}}_{\left(g_{1}, \ldots, g_{n}\right)}$ and $c_{1} \otimes \ldots \otimes c_{n} \in \mathcal{C}$ and for all $g=\left(g_{1}, \ldots, g_{n}\right) \in \overline{\mathbf{G}}$.

(2) It remains to prove that

$$
\tilde{\mathbf{M}} \otimes_{\mathbf{A}^{\prime}}(\tilde{\mathbf{M}})^{*} \simeq \mathbf{A} \text { as } \overline{\mathbf{G}} \text {-graded }(\mathbf{A}, \mathbf{A}) \text {-bimodules over } \mathcal{C},
$$

and that

$$
(\tilde{\mathbf{M}})^{*} \otimes_{\mathbf{A}} \tilde{\mathbf{M}} \simeq \mathbf{A}^{\prime} \text { as } \overline{\mathbf{G}} \text {-graded }\left(\mathbf{A}^{\prime}, \mathbf{A}^{\prime}\right) \text {-bimodules over } \mathcal{C} .
$$

We will only check the first isomorphism:

$$
\begin{aligned}
\tilde{\mathbf{M}} \otimes \mathbf{A}^{\prime}(\tilde{\mathbf{M}})^{*}= & \left(\tilde{M}_{1} \otimes \ldots \otimes \tilde{M}_{n}\right) \otimes_{\mathbf{A}^{\prime}}\left(\tilde{M}_{1} \otimes \ldots \otimes \tilde{M}_{n}\right)^{*} \\
\simeq & \left(\tilde{M}_{1} \otimes \ldots \otimes \tilde{M}_{n}\right) \otimes_{\mathbf{A}^{\prime}}\left(\tilde{M}_{1}^{*} \otimes \ldots \otimes \tilde{M}_{n}^{*}\right) \\
= & \left(\tilde{M}_{1} \otimes \ldots \otimes \tilde{M}_{n}\right) \otimes_{A_{1}^{\prime} \otimes \ldots \otimes A_{n}^{\prime}}\left(\tilde{M}_{1}^{*} \otimes \ldots \otimes \tilde{M}_{n}^{*}\right) \\
\simeq & \left(\tilde{M}_{1} \otimes \tilde{A}_{1}^{\prime} \tilde{M}_{1}^{*}\right) \otimes \ldots \otimes\left(\tilde{M}_{n} \otimes A_{n}^{\prime} \tilde{M}_{n}^{*}\right) \\
\simeq & A_{1} \otimes \ldots \otimes A_{n}=\mathbf{A},
\end{aligned}
$$

as $\overline{\mathbf{G}}$-graded (A, A)-bimodules over $\mathcal{C}$. 


\section{Wreath products for algebras}

Consider the notations from Section 2 . We denote $\bar{G}^{n}:=\bar{G} \times \ldots \times \bar{G}(n$ times $)$. We recall the definition of a wreath product as in [7, Definition 2.19] and [1, Section 5.1.C]:

Definition 4.1. The wreath product $\bar{G} \curlyvee S_{n}$ is the semidirect product $\bar{G}^{n} \rtimes S_{n}$, where $S_{n}$ acts on $\bar{G}^{n}$ (on the left) by permuting the components:

$$
{ }^{\sigma}\left(g_{1}, \ldots, g_{n}\right):=\left(g_{\sigma^{-1}(1)}, \ldots, g_{\sigma^{-1}(n)}\right)
$$

More exactly, the elements of $\bar{G} \prec S_{n}$ are of the form $\left(\left(g_{1}, \ldots, g_{n}\right), \sigma\right)$, and the multiplication is:

$$
\left(\left(g_{1}, \ldots, g_{n}\right), \sigma\right)\left(\left(h_{1}, \ldots, h_{n}\right), \tau\right):=\left(\left(g_{1}, \ldots, g_{n}\right) \cdot{ }^{\sigma}\left(h_{1}, \ldots, h_{n}\right), \sigma \tau\right),
$$

for all $g_{1}, \ldots, g_{n}, h_{1}, \ldots, h_{n} \in \bar{G}$ and $\sigma, \tau \in S_{n}$.

Definition 4.2. Let $A$ be an algebra. We denote by $A^{\otimes n}:=A \otimes \ldots \otimes A$ ( $n$ times). The wreath product $A$ ? $S_{n}$ is

$$
A \curlywedge S_{n}:=A^{\otimes n} \otimes \mathcal{O} S_{n}
$$

as $\mathcal{O}$-modules, with multiplication

$$
\begin{aligned}
& \left(\left(a_{1} \otimes \ldots \otimes a_{n}\right) \otimes \sigma\right)\left(\left(b_{1} \otimes \ldots \otimes b_{n}\right) \otimes \tau\right) \\
& \quad:=\left(\left(a_{1} \otimes \ldots \otimes a_{n}\right) \cdot \sigma\left(b_{1} \otimes \ldots \otimes b_{n}\right)\right) \otimes(\sigma \tau),
\end{aligned}
$$

where

$$
\sigma\left(b_{1} \otimes \ldots \otimes b_{n}\right):=b_{\sigma^{-1}(1)} \otimes \ldots \otimes b_{\sigma^{-1}(n)},
$$

for all $\left(a_{1} \otimes \ldots \otimes a_{n}\right) \otimes \sigma,\left(b_{1} \otimes \ldots \otimes b_{n}\right) \otimes \tau \in A \zeta S_{n}$.

Lemma 4.3. Let $A$ be a $\bar{G}$-graded algebra and $\mathcal{C}$ be a $\bar{G}$-graded $\bar{G}$-acted algebra. The following affirmations hold:

(1) $A$ < $S_{n}$ is a $\bar{G}$ 々 $S_{n}$-graded algebra;

(2) If $A$ is a $\bar{G}$-graded crossed product, then $A \backslash S_{n}$ is a $\bar{G} \backslash S_{n}$-graded crossed product;

(3) $\mathcal{C}^{\otimes n}$ is a $\bar{G} ₹ S_{n}$-acted $\bar{G}^{n}$-graded algebra;

(4) If $A$ is a $\bar{G}$-graded algebra over $\mathcal{C}$, then $A \backslash S_{n}$ is a $\bar{G} \backslash S_{n}$-graded algebra over $\mathcal{C}^{\otimes n}$.

Proof. (1) The $\left(\left(g_{1}, \ldots, g_{n}\right), \sigma\right)$-component of $A$ < $S_{n}$ is

$$
\left(A \succ S_{n}\right)_{\left(\left(g_{1}, \ldots, g_{n}\right), \sigma\right)}:=\left(A_{g_{1}} \otimes \ldots \otimes A_{g_{n}}\right) \otimes \mathcal{O} \sigma
$$

for each $\left(\left(g_{1}, \ldots, g_{n}\right), \sigma\right) \in \bar{G} \prec S_{n}$. Indeed,

$$
\begin{aligned}
& \left(A \prec S_{n}\right)_{\left(\left(g_{1}, \ldots, g_{n}\right), \sigma\right)}\left(A \prec S_{n}\right)_{\left(\left(h_{1}, \ldots, h_{n}\right), \tau\right)} \\
& =\left(\left(A_{g_{1}} \otimes \ldots \otimes A_{g_{n}}\right) \otimes \mathcal{O} \sigma\right)\left(\left(A_{h_{1}} \otimes \ldots \otimes A_{h_{n}}\right) \otimes \mathcal{O} \tau\right) \\
& =\left(\left(A_{g_{1}} \otimes \ldots \otimes A_{g_{n}}\right) \cdot{ }^{\sigma}\left(A_{h_{1}} \otimes \ldots \otimes A_{h_{n}}\right)\right) \otimes(\mathcal{O} \sigma \otimes \mathcal{O} \tau) \\
& =\left(\left(A_{g_{1}} \otimes \ldots \otimes A_{g_{n}}\right) \cdot\left(A_{h_{\sigma^{-1}(1)}} \otimes \ldots \otimes A_{h_{\sigma^{-1}(n)}}\right)\right) \otimes \mathcal{O}(\sigma \tau) \\
& =\left(A_{g_{1}} A_{h_{\sigma^{-1}(1)}} \otimes \ldots \otimes A_{g_{n}} A_{h_{\sigma^{-1}(n)}}\right) \otimes \mathcal{O}(\sigma \tau) \\
& \subseteq\left(A_{g_{1} h_{\sigma^{-1}(1)}} \otimes \ldots \otimes A_{g_{n} h_{\sigma^{-1}(n)}}\right) \otimes \mathcal{O}(\sigma \tau) \\
& =\left(A \nmid S_{n}\right)_{\left(\left(\left(g_{1}, \ldots, g_{n}\right) \cdot \sigma\left(h_{1}, \ldots, h_{n}\right)\right), \sigma \tau\right)}
\end{aligned}
$$




$$
=\left(A \curlywedge S_{n}\right)_{\left(\left(g_{1}, \ldots, g_{n}\right), \sigma\right)\left(\left(h_{1}, \ldots, h_{n}\right), \tau\right)} .
$$

(2) We choose invertible homogeneous elements $u_{g} \in A_{g}$ for all $g \in \bar{G}$. For $\left(\left(g_{1}, \ldots, g_{n}\right), \sigma\right) \in \bar{G} \prec S_{n}$ the homogeneous element

$$
u_{\left(\left(g_{1}, \ldots, g_{n}\right), \sigma\right)}:=\left(u_{g_{1}} \otimes \ldots \otimes u_{g_{n}}\right) \otimes \sigma,
$$

is clearly invertible, with

$$
u_{\left(\left(g_{1}, \ldots, g_{n}\right), \sigma\right)}^{-1}:=\left(u_{g_{\sigma(1)}}^{-1} \otimes \ldots \otimes u_{g_{\sigma(n)}}^{-1}\right) \otimes \sigma^{-1} .
$$

(3) By Lemma 3.2, we know that $\mathcal{C}^{\otimes n}$ is a $\bar{G}^{n}$-graded algebra. It remains to prove that it is $\bar{G}\left\{S_{n}\right.$-acted and that the action is compatible with the gradings. We define the action of $\bar{G} \imath S_{n}$ on $\mathcal{C}^{\otimes n}$ as follows:

$$
\left(\left(g_{1}, \ldots, g_{n}\right), \sigma\right)\left(c_{1} \otimes \ldots \otimes c_{n}\right):={ }^{g_{1}} c_{\sigma^{-1}(1)} \otimes \ldots \otimes{ }^{g_{n}} c_{\sigma^{-1}(n)},
$$

where $\left(\left(g_{1}, \ldots, g_{n}\right), \sigma\right) \in \bar{G} \imath S_{n}$ and $c_{1} \otimes \ldots \otimes c_{n} \in \mathcal{C}^{\otimes n}$. We have:

$$
\begin{aligned}
& { }^{\left(\left(1_{\bar{G}}, \ldots, 1_{\bar{G}}\right), e\right)}\left(a_{1} \otimes \ldots \otimes a_{n}\right)={ }^{1_{\bar{G}}} a_{1} \otimes \ldots \otimes{ }^{1_{\bar{G}}} a_{n} \\
& =a_{1} \otimes \ldots \otimes a_{n} \text {, } \\
& \left(\left(\left(g_{1}, \ldots, g_{n}\right), \sigma\right)\left(\left(h_{1}, \ldots, h_{n}\right), \tau\right)\right)\left(a_{1} \otimes \ldots \otimes a_{n}\right) \\
& ={ }^{\left(\left(g_{1}, \ldots, g_{n}\right) \cdot{ }^{\sigma}\left(h_{1}, \ldots, h_{n}\right), \sigma \tau\right)}\left(a_{1} \otimes \ldots \otimes a_{n}\right) \\
& =\left(\left(g_{1}, \ldots, g_{n}\right) \cdot\left(h_{\sigma^{-1}(1)}, \ldots, h_{\sigma^{-1}(n)}\right), \sigma \tau\right)\left(a_{1} \otimes \ldots \otimes a_{n}\right) \\
& ={ }^{\left(\left(g_{1} h_{\sigma^{-1}(1)}, \ldots, g_{n} h_{\sigma^{-1}(n)}\right), \sigma \tau\right)}\left(a_{1} \otimes \ldots \otimes a_{n}\right) \\
& ={ }^{g_{1} h_{\sigma^{-1}(1)}} a_{(\sigma \tau)^{-1}(1)} \otimes \ldots \otimes{ }^{g_{n} h_{\sigma^{-1}(n)}} a_{(\sigma \tau)^{-1}(n)} \\
& ={ }^{g_{1} h_{\sigma^{-1}(1)}} a_{\tau^{-1}\left(\sigma^{-1}(1)\right)} \otimes \ldots \otimes \otimes^{g_{n} h_{\sigma^{-1}(n)}} a_{\tau^{-1}\left(\sigma^{-1}(n)\right)} \\
& ={ }^{\left(\left(g_{1}, \ldots, g_{n}\right), \sigma\right)}\left({ }^{h_{1}} a_{\tau^{-1}(1)} \otimes \ldots \otimes{ }^{h_{n}} a_{\tau^{-1}(n)}\right) \\
& ={ }^{\left(\left(g_{1}, \ldots, g_{n}\right), \sigma\right)}\left(\left(\left(h_{1}, \ldots, h_{n}\right), \tau\right)\left(a_{1} \otimes \ldots \otimes a_{n}\right)\right) \text {, } \\
& \left(\left(g_{1}, \ldots, g_{n}\right), \sigma\right)\left(\left(a_{1} \otimes \ldots \otimes a_{n}\right) \cdot\left(b_{1} \otimes \ldots \otimes b_{n}\right)\right) \\
& ={ }^{\left(\left(g_{1}, \ldots, g_{n}\right), \sigma\right)}\left(a_{1} b_{1} \otimes \ldots \otimes a_{n} b_{n}\right) \\
& ={ }^{g_{1}}\left(a_{\sigma^{-1}(1)} b_{\sigma^{-1}(1)}\right) \otimes \ldots \otimes{ }^{g_{n}}\left(a_{\sigma^{-1}(n)} b_{\sigma^{-1}(n)}\right) \\
& ={ }^{g_{1}} a_{\sigma^{-1}(1)} \cdot{ }^{g_{1}} b_{\sigma^{-1}(1)} \otimes \ldots \otimes{ }^{g_{n}} a_{\sigma^{-1}(n)} \cdot{ }^{g_{n}} b_{\sigma^{-1}(n)} \\
& =\left({ }^{g_{1}} a_{\sigma^{-1}(1)} \otimes \ldots \otimes{ }^{g_{n}} a_{\sigma^{-1}(n)}\right)\left({ }^{g_{1}} b_{\sigma^{-1}(1)} \otimes \ldots \otimes{ }^{g_{n}} b_{\sigma^{-1}(n)}\right) \\
& ={ }^{\left(\left(g_{1}, \ldots, g_{n}\right), \sigma\right)}\left(a_{1} \otimes \ldots \otimes a_{n}\right) \cdot{ }^{\left(\left(g_{1}, \ldots, g_{n}\right), \sigma\right)}\left(b_{1} \otimes \ldots \otimes b_{n}\right) \text {, }
\end{aligned}
$$

and

$$
\begin{aligned}
& { }^{\left(\left(h_{1}, \ldots, h_{n}\right), \tau\right)}\left(c_{1} \otimes \ldots \otimes c_{n}\right)={ }^{h_{1}} c_{\tau^{-1}(1)} \otimes \ldots \otimes{ }^{h_{n}} c_{\tau^{-1}(n)} \\
& \in \mathcal{C}_{h_{1} g_{\tau-1}(1)} \otimes \ldots \otimes \mathcal{C}_{h_{n} g_{\tau-1}(n)}
\end{aligned}
$$

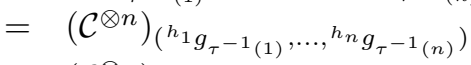

$$
\begin{aligned}
& =\left(\mathcal{C}^{\otimes n}\right)_{\left(\left(h_{1}, \ldots, h_{n}\right), \tau\right)}\left(g_{1}, \ldots, g_{n}\right),
\end{aligned}
$$

for all $a_{1} \otimes \ldots \otimes a_{n}, b_{1} \otimes \ldots \otimes b_{n} \in \mathcal{C}^{\otimes n}$, for all $c_{1} \otimes \ldots \otimes c_{n} \in \mathcal{C}_{\left(g_{1}, \ldots, g_{n}\right)}^{\otimes n}$ and for all $\left(\left(g_{1}, \ldots, g_{n}\right), \sigma\right),\left(\left(h_{1}, \ldots, h_{n}\right), \tau\right) \in \bar{G} \curlywedge S_{n}$. 
(4) By assumption, there exists a $\bar{G}$-graded $\bar{G}$-acted algebra homomorphism $\zeta: \mathcal{C} \rightarrow C_{A}(B)$, where $B$ is the identity component of $A$. Henceforth, we have a $\bar{G}^{n}$-graded $\bar{G}^{n}$-acted algebra homomorphism:

$$
\zeta^{\otimes n}: \mathcal{C}^{\otimes n} \rightarrow C_{A}(B)^{\otimes n}
$$

Now, via the identification:

$$
A^{\otimes n} \ni a_{1} \otimes \ldots \otimes a_{n}=\left(a_{1} \otimes \ldots \otimes a_{n}\right) \otimes e \in A \curlywedge S_{n},
$$

we clearly have the following inclusion:

$$
C_{A}(B)^{\otimes n} \subseteq C_{A \imath S_{n}}\left(B^{\otimes n}\right)=C_{A \imath S_{n}}\left(\left(A \succ S_{n}\right)_{\left(\left(1_{\bar{G}}, \ldots, 1_{\bar{G}}\right), e\right)}\right) .
$$

Therefore, we obtain the required $\bar{G} \prec S_{n}$-graded $\bar{G} \imath S_{n}$-acted algebra map

$$
\zeta_{\mathrm{wr}}: \mathcal{C}^{\otimes n} \rightarrow C_{A 2 S_{n}}\left(\left(A \curlywedge S_{n}\right)_{\left(\left(1_{\bar{G}}, \ldots, 1_{\bar{G}}\right), e\right)}\right) .
$$

Indeed, given $\left(\left(g_{1}, \ldots, g_{n}\right), \sigma\right) \in \bar{G} \curlywedge S_{n}$ and $c_{1} \otimes \ldots \otimes c_{n} \in \mathcal{C}^{\otimes n}$ we have:

$$
\begin{aligned}
\zeta_{\mathrm{wr}}\left(\left({ }^{\left.\left(g_{1}, \ldots, g_{n}\right), \sigma\right)}\left(c_{1} \otimes \ldots \otimes c_{n}\right)\right)\right. & =\zeta_{\mathrm{wr}}\left({ }^{g_{1}} c_{\sigma^{-1}}(1) \otimes \ldots \otimes{ }^{g_{n}} c_{\sigma^{-1}(n)}\right) \\
& =\zeta\left({ }^{g_{1}} c_{\sigma^{-1}(1)}\right) \otimes \ldots \otimes \zeta\left({ }^{g_{n}} c_{\sigma^{-1}(n)}\right) \\
& ={ }^{g_{1}} \zeta\left(c_{\sigma^{-1}(1)}\right) \otimes \ldots \otimes{ }^{g_{n}} \zeta\left(c_{\sigma^{-1}(n)}\right) \\
& ={ }^{\left(\left(g_{1}, \ldots, g_{n}\right), \sigma\right)}\left(\zeta\left(c_{1}\right) \otimes \ldots \otimes \zeta\left(c_{n}\right)\right) \\
& ={ }^{\left(\left(g_{1}, \ldots, g_{n}\right), \sigma\right)} \zeta_{\mathrm{wr}}\left(c_{1} \otimes \ldots \otimes c_{n}\right) .
\end{aligned}
$$

Henceforth, $A \nmid S_{n}$ is a $\bar{G} \nmid S_{n}$-graded algebra over $\mathcal{C}^{\otimes n}$.

\section{Morita equivalences for wreath products}

Consider the notations from Section 2 and Section 4. We recall the definition of a wreath product between a module and $S_{n}$.

Definition 5.1. Let $A$ and $A^{\prime}$ be two algebras. Assume that $\tilde{M}$ is an $\left(A, A^{\prime}\right)$-bimodule. The wreath product $\tilde{M}<S_{n}$ is defined by

$$
\tilde{M} \succ S_{n}:=\tilde{M}^{\otimes n} \otimes \mathcal{O} S_{n}
$$

as $\mathcal{O}$-modules, with operations

$$
\begin{aligned}
& \left(\left(a_{1} \otimes \ldots \otimes a_{n}\right) \otimes \sigma\right)\left(\left(\tilde{m}_{1} \otimes \ldots \otimes \tilde{m}_{n}\right) \otimes \tau\right) \\
& \quad:=\left(\left(a_{1} \otimes \ldots \otimes a_{n}\right) \cdot{ }^{\sigma}\left(\tilde{m}_{1} \otimes \ldots \otimes \tilde{m}_{n}\right)\right) \otimes(\sigma \tau),
\end{aligned}
$$

and

$$
\begin{aligned}
& \left(\left(\tilde{m}_{1} \otimes \ldots \otimes \tilde{m}_{n}\right) \otimes \tau\right)\left(\left(a_{1}^{\prime} \otimes \ldots \otimes a_{n}^{\prime}\right) \otimes \pi\right) \\
& \quad:=\left(\left(\tilde{m}_{1} \otimes \ldots \otimes \tilde{m}_{n}\right) \cdot \tau\left(a_{1}^{\prime} \otimes \ldots \otimes a_{n}^{\prime}\right)\right) \otimes(\tau \pi),
\end{aligned}
$$

where

$$
{ }^{\sigma}\left(\tilde{m}_{1} \otimes \ldots \otimes \tilde{m}_{n}\right):=\tilde{m}_{\sigma^{-1}(1)} \otimes \ldots \otimes \tilde{m}_{\sigma^{-1}(n)},
$$

for all $\left(a_{1} \otimes \ldots \otimes a_{n}\right) \otimes \sigma \in A 2 S_{n},\left(\tilde{m}_{1} \otimes \ldots \otimes \tilde{m}_{n}\right) \otimes \tau \in \tilde{M} 2 S_{n}$ and $\left(a_{1}^{\prime} \otimes \ldots \otimes a_{n}^{\prime}\right) \otimes \pi \in$ $A^{\prime} \curlyvee S_{n}$. 
5.2. Let $\mathcal{C}$ be a $\bar{G}$-graded $\bar{G}$-acted algebra and $A$ and $A^{\prime}$ be two $\bar{G}$-graded crossed products over $\mathcal{C}$, with identity components $B$ and $B^{\prime}$ respectively.

If $\tilde{M}$ is an $\left(A, A^{\prime}\right)$-bimodule which induces a Morita equivalence between $A$ and $A^{\prime}$, by the results of [1, Section 5.1.C], we already know that $\tilde{M}<S_{n}$ induces a Morita equivalence between $A$ < $S_{n}$ and $A^{\prime}$ 々 $S_{n}$. The question that arises is whether this result can be extended to give a graded Morita equivalence over a group graded group acted algebra.

Theorem 5.3. Let $\tilde{M}$ be a $\bar{G}$-graded $\left(A, A^{\prime}\right)$-bimodule over $\mathcal{C}$. Then, the following affirmations hold:

(1) $\tilde{M} \backslash S_{n}$ is a $\bar{G} \backslash S_{n}$-graded $\left(A<S_{n}, A^{\prime} \curlyvee S_{n}\right)$-bimodule over $\mathcal{C}^{\otimes n}$;

(2) $\left(A \imath S_{n}\right) \otimes_{B^{\otimes n}} M^{\otimes n} \simeq M^{\otimes n} \otimes_{B^{\prime \otimes n}}\left(A^{\prime} \imath S_{n}\right) \simeq \tilde{M} 2 S_{n}$ as $\bar{G}\left\{S_{n}\right.$-graded $\left(A 2 S_{n}, A^{\prime} 2 S_{n}\right)$ bimodules over $\mathcal{C}^{\otimes n}$, where $M$ is the identity component of $\tilde{M}$;

(3) If $\tilde{M}$ induces a $\bar{G}$-graded Morita equivalence over $\mathcal{C}$ between $A$ and $A^{\prime}$, then $\tilde{M} \supsetneq S_{n}$ induces a $\bar{G} \supsetneq S_{n}$-graded Morita equivalence over $\mathcal{C}^{\otimes n}$ between $A \prec S_{n}$ and $A^{\prime} \chi S_{n}$.

Proof. (1) By Lemma 4.3, we know that $A \curlywedge S_{n}$ and $A^{\prime} 々 S_{n}$ are $\bar{G} 々 S_{n}$-graded crossed products over $\mathcal{C}^{\otimes n}$.

It is also known that $A<S_{n}$ and $A^{\prime} \curlyvee S_{n}$ are strongly $S_{n}$-graded algebras, and given this grading, if we denote

$$
\Delta_{S_{n}}\left(A \prec S_{n} \otimes\left(A^{\prime} \succ S_{n}\right)^{\mathrm{op}}\right):=\left(A \curlywedge S_{n} \otimes\left(A^{\prime} \succ S_{n}\right)^{\mathrm{op}}\right)_{\delta\left(S_{n}\right)},
$$

where $\delta\left(S_{n}\right):=\left\{\left(\sigma, \sigma^{-1}\right) \mid \sigma \in S_{n}\right\}$, we have the following isomorphism of algebras:

$$
\Delta_{S_{n}}\left(A \succ S_{n} \otimes\left(A^{\prime} \succ S_{n}\right)^{\mathrm{op}}\right) \simeq\left(A^{\otimes n} \otimes\left(A^{\prime \otimes n}\right)^{\mathrm{op}}\right) \otimes \mathcal{O} S_{n}
$$

Moreover, [1, Lemma 5.1.19] states that $\tilde{M}^{\otimes n}$ is a left $\mathcal{O} S_{n}$-module with the action given by permutations. Henceforth, it is easy to see that $\tilde{M}^{\otimes n}$ is a $\left(A^{\otimes n} \otimes\left(A^{\prime \otimes n}\right)^{\mathrm{op}}\right) \otimes$ $\mathcal{O} S_{n}$-module, and thereby (the above isomorphism), $\tilde{M}^{\otimes n}$ extends to a $\Delta_{S_{n}}\left(A \succ S_{n} \otimes\right.$ $\left(A^{\prime}\left(S_{n}\right)^{\text {op }}\right)$-module. Thus, $\tilde{M}<S_{n}:=\tilde{M}^{\otimes n} \otimes \mathcal{O} S_{n}$ becomes an $\left(A 2 S_{n}, A^{\prime} \prec S_{n}\right)$-bimodule.

Now, we will prove that $\tilde{M} \prec S_{n}$ is a $\bar{G} \curlyvee S_{n}$-graded $\left(A<S_{n}, A^{\prime} \curlyvee S_{n}\right)$-bimodule. Indeed, for all $\left(\left(g_{1}, \ldots, g_{n}\right), \sigma\right) \in \bar{G} \prec S_{n}$, the $\left(\left(g_{1}, \ldots, g_{n}\right), \sigma\right)$-component of $\tilde{M} \prec S_{n}$ is:

$$
\left(\tilde{M} \succ S_{n}\right)_{\left(\left(g_{1}, \ldots, g_{n}\right), \sigma\right)}:=\left(\tilde{M}_{g_{1}} \otimes \ldots \otimes \tilde{M}_{g_{n}}\right) \otimes \mathcal{O} \sigma .
$$

The verification for this definition is straightforward, as follows. For each $\left(\left(g_{1}, \ldots, g_{n}\right), \sigma\right),\left(\left(x_{1}, \ldots, x_{n}\right), \pi\right)$ and $\left(\left(h_{1}, \ldots, h_{n}\right), \tau\right) \in \bar{G} \curlywedge S_{n}$ we have:

$\left(A \succ S_{n}\right)_{\left(\left(g_{1}, \ldots, g_{n}\right), \sigma\right)}\left(\tilde{M}<S_{n}\right)_{\left(\left(x_{1}, \ldots, x_{n}\right), \pi\right)}\left(A^{\prime} \prec S_{n}\right)_{\left(\left(h_{1}, \ldots, h_{n}\right), \tau\right)}$

$=\left(\left(A_{g_{1}} \otimes \ldots \otimes A_{g_{n}}\right) \otimes \mathcal{O} \sigma\right)\left(\left(\tilde{M}_{x_{1}} \otimes \ldots \otimes \tilde{M}_{x_{n}}\right) \otimes \mathcal{O} \pi\right)\left(\left(A_{h_{1}}^{\prime} \otimes \ldots \otimes A_{h_{n}}^{\prime}\right) \otimes \mathcal{O} \tau\right)$

$=\left(\left(A_{g_{1}} \tilde{M}_{x_{\sigma^{-1}(1)}} \otimes \ldots \otimes A_{g_{n}} \tilde{M}_{x_{\sigma^{-1}(n)}}\right) \otimes \mathcal{O}(\sigma \pi)\right)\left(\left(A_{h_{1}}^{\prime} \otimes \ldots \otimes A_{h_{n}}^{\prime}\right) \otimes \mathcal{O} \tau\right)$

$=\left(A_{g_{1}} \tilde{M}_{x_{\sigma^{-1}(1)}} A_{h_{(\sigma \pi)^{-1}(1)}^{\prime}}^{\prime} \otimes \ldots \otimes A_{g_{n}} \tilde{M}_{x_{\sigma^{-1}(n)}} A_{h_{(\sigma \pi)-1}(n)}^{\prime}\right) \otimes \mathcal{O}(\sigma \pi \tau)$

$\subseteq\left(\tilde{M}_{g_{1} x_{\sigma^{-1}(1)} h_{(\sigma \pi)^{-1}(1)}} \otimes \ldots \otimes \tilde{M}_{g_{n} x_{\sigma^{-1}(n)} h_{(\sigma \pi)^{-1}(n)}}\right) \otimes \mathcal{O}(\sigma \pi \tau)$

$=\left(\tilde{M} \backslash S_{n}\right)_{\left(\left(g_{1} x_{\sigma^{-1}(1)} h_{(\sigma \pi)-1(1)}, \ldots, g_{n} x_{\sigma^{-1}(n)} h_{(\sigma \pi)-1(n)}\right), \sigma \pi \tau\right)}$

$=\left(\tilde{M} \succ S_{n}\right)_{\left(\left(g_{1} x_{\sigma-1(1)}, \ldots, g_{n} x_{\sigma-1(n)}\right), \sigma \pi\right)\left(\left(h_{1}, \ldots, h_{n}\right), \tau\right)}$

$=\left(\tilde{M} \succ S_{n}\right)_{\left(\left(g_{1}, \ldots, g_{n}\right), \sigma\right)\left(\left(x_{1}, \ldots, x_{n}\right), \pi\right)\left(\left(h_{1}, \ldots, h_{n}\right), \tau\right)}$. 
Therefore, $\tilde{M} \prec S_{n}$ is a $\bar{G} \prec S_{n}$-graded $\left(A<S_{n}, A^{\prime} \prec S_{n}\right)$-bimodule. Note that the identity component of $\tilde{M}<S_{n}$ (with respect to the $\bar{G}<S_{n}$-grading) is

$$
\left(\tilde{M}<S_{n}\right)_{1}=M^{\otimes n},
$$

where $M$ is the identity component of $\tilde{M}$.

Finally, we will prove that

$$
\begin{aligned}
\left(\left(\tilde{m}_{g_{1}} \otimes \ldots \otimes \tilde{m}_{g_{n}}\right) \otimes \sigma\right)\left(c_{1} \otimes \ldots \otimes c_{n}\right) & \\
& ={ }^{\left(\left(g_{1}, \ldots, g_{n}\right), \sigma\right)}\left(c_{1} \otimes \ldots \otimes c_{n}\right)\left(\left(\tilde{m}_{g_{1}} \otimes \ldots \otimes \tilde{m}_{g_{n}}\right) \otimes \sigma\right),
\end{aligned}
$$

for all $\left(\tilde{m}_{g_{1}} \otimes \ldots \otimes \tilde{m}_{g_{n}}\right) \otimes \sigma \in\left(\tilde{M} 乙 S_{n}\right)_{\left(\left(g_{1}, \ldots, g_{n}\right), \sigma\right)}$ and $c_{1} \otimes \ldots \otimes c_{n} \in \mathcal{C} \otimes n$ and for all $\left(\left(g_{1}, \ldots, g_{n}\right), \sigma\right) \in \bar{G} \succ S_{n}$. Indeed,

$$
\begin{aligned}
& \left(\left(\tilde{m}_{g_{1}} \otimes \ldots \otimes \tilde{m}_{g_{n}}\right) \otimes \sigma\right)\left(c_{1} \otimes \ldots \otimes c_{n}\right) \\
& \quad=\left(\tilde{m}_{g_{1}} c_{\sigma^{-1}(1)} \otimes \ldots \otimes \tilde{m}_{g_{n}} c_{\sigma^{-1}(n)}\right) \otimes \sigma \\
& \quad=\left({ }^{{ }^{1}} c_{\sigma^{-1}(1)} \tilde{m}_{g_{1}} \otimes \ldots \otimes{ }^{g_{n}} c_{\sigma^{-1}(n)} \tilde{m}_{g_{n}}\right) \otimes \sigma \\
& \quad=\left({ }^{{ }^{1}} c_{\sigma^{-1}(1)} \otimes \ldots \otimes{ }^{g_{n}} c_{\sigma^{-1}(n)}\right)\left(\left(\tilde{m}_{g_{1}} \otimes \ldots \otimes \tilde{m}_{g_{n}}\right) \otimes \sigma\right) \\
& \quad=\left(\left({ }^{\left.\left(g_{1}, \ldots, g_{n}\right), \sigma\right)}\left(c_{1} \otimes \ldots \otimes c_{n}\right)\left(\left(\tilde{m}_{g_{1}} \otimes \ldots \otimes \tilde{m}_{g_{n}}\right) \otimes \sigma\right) .\right.\right.
\end{aligned}
$$

Henceforth, $\tilde{M}<S_{n}$ is a $\bar{G} \succ S_{n}$-graded $\left(A \succ S_{n}, A^{\prime} \succ S_{n}\right)$-bimodule over $\mathcal{C}^{\otimes n}$.

(2) In this part, in order to prove our claim, we want to use a similar technique as in part (1), but with regard to the grading given by $\bar{G}$ ? $S_{n}$.

Henceforth, we regard $\left(A^{\prime} \succ S_{n}\right)^{\text {op }}$ as a $\bar{G} \succ S_{n}$-graded crossed product over $\mathcal{C}^{\otimes n}$, where the $\left(\left(g_{1}, \ldots, g_{n}\right), \sigma\right)$-component of $\left(A^{\prime}<S_{n}\right)^{\text {op }}$ is:

$$
\left(A^{\prime} \prec S_{n}\right)_{\left(\left(g_{1}, \ldots, g_{n}\right), \sigma\right)}^{\mathrm{op}}:=\left(\left(A^{\prime} \prec S_{n}\right)_{\left(\left(g_{1}, \ldots, g_{n}\right), \sigma\right)^{-1}}\right)^{\mathrm{op}},
$$

for all $\left(\left(g_{1}, \ldots, g_{n}\right), \sigma\right) \in \bar{G} \curlywedge S_{n}$, and we consider:

$$
\begin{aligned}
& \Delta_{\bar{G}\left(S_{n}\right.}^{\mathcal{C} \otimes n}\left(A<S_{n} \otimes_{\mathcal{C} \otimes n}\left(A^{\prime}<S_{n}\right)^{\mathrm{op}}\right):= \\
& \bigoplus_{\left(\left(g_{1}, \ldots, g_{n}\right), \sigma\right) \in \bar{G} l S_{n}}\left(\left(A \succ S_{n}\right)_{\left(\left(g_{1}, \ldots, g_{n}\right), \sigma\right)} \otimes_{\mathcal{C} \otimes n}\left(A^{\prime} \zeta S_{n}\right)_{\left(\left(g_{1}, \ldots, g_{n}\right), \sigma\right)}^{\mathrm{op}}\right),
\end{aligned}
$$

which, by [3, Lemma 2.8], is an $\mathcal{O}$-algebra.

Now, given the fact from part (1) of this theorem, that $\tilde{M}\urcorner S_{n}$ is a $\bar{G} \curlyvee S_{n^{-}}$ graded $\left(A<S_{n}, A^{\prime} \succ S_{n}\right)$-bimodule over $\mathcal{C}^{\otimes n}$, we obtain, by [3, Proposition 2.11], that $\left(\tilde{M} 々 S_{n}\right)_{1}=M^{\otimes n}$ extends to a $\Delta_{\bar{G}\left(S_{n}\right.}^{\mathcal{C}^{\otimes n}}\left(A<S_{n} \otimes_{\mathcal{C} \otimes n}\left(A^{\prime} \succ S_{n}\right)^{\text {op }}\right)$-module and that we have the following isomorphisms of $\bar{G} \imath S_{n}$-graded $\left(A \prec S_{n}, A^{\prime} \imath S_{n}\right)$-bimodules over $\mathcal{C}^{\otimes n}$ :

$$
\left(A \succ S_{n}\right) \otimes_{B^{\otimes n}} M^{\otimes n} \simeq M^{\otimes n} \otimes_{B^{\prime} \otimes n}\left(A^{\prime} \curlyvee S_{n}\right) \simeq \tilde{M}\left\ulcorner S_{n} .\right.
$$

More exactly, these isomorphisms are:

$$
f:\left(A \succ S_{n}\right) \otimes_{B \otimes n} M^{\otimes n} \rightarrow \tilde{M}\left\ulcorner S_{n}, \quad(a \otimes \sigma) \otimes m \mapsto\left(a \cdot{ }^{\sigma} m\right) \otimes \sigma,\right.
$$

and

$$
g: M^{\otimes n} \otimes_{B^{\prime \otimes n}}\left(A^{\prime} 々 S_{n}\right) \rightarrow \tilde{M} 々 S_{n}, \quad m \otimes\left(a^{\prime} \otimes \sigma\right) \mapsto\left(m \cdot a^{\prime}\right) \otimes \sigma
$$

for all $a \in A^{\otimes n}, a^{\prime} \in A^{\prime \otimes n}, m \in M^{\otimes n}$ and $\sigma \in S_{n}$.

We prove that $f$ is an isomorphism of $\bar{G}<S_{n}$-graded $\left(A<S_{n}, A^{\prime}<S_{n}\right.$ )-bimodules over $\mathcal{C}^{\otimes n}$. The verification for $g$ is similar. The left $A$ < $S_{n}$-module structure of $(A$ ) 
$\left.S_{n}\right) \otimes_{B} \otimes n M^{\otimes n}$ is clear. We recall and particularize from [3, Proposition 2.11] the right $A^{\prime} \curlyvee S_{n}$-module structure of $\left(A \succ S_{n}\right) \otimes_{B \otimes n} M^{\otimes n}$ :

$$
\begin{aligned}
((a & \otimes \sigma) \otimes_{B} \otimes n \\
& =\left((a \otimes \sigma)\left(u_{g} \otimes \tau\right)\right) \otimes_{B} \otimes n \\
& =\left(\left(a^{\prime} \cdot{ }^{\sigma} u_{g} \otimes \sigma \tau\right) \otimes_{B} \otimes n\right. \\
& \left(\left(\left(^{\tau^{-1}} u_{g}^{-1} \otimes \tau^{-1}\right)(m \otimes e)\left(a_{g}^{\prime} \otimes \tau\right)\right)\right. \\
& =\left(a \cdot{ }^{\sigma} u_{g} \otimes \sigma \tau\right) \otimes_{B \otimes n}\left(\left({ }^{-1} u_{g}^{-1} \cdot{ }^{-1} m \otimes \tau^{-1}\right)\left(a_{g}^{\prime} \otimes \tau\right)\right) \\
& =\left(a \cdot{ }^{\sigma} u_{g} \otimes \sigma \tau\right) \otimes_{B} \otimes n \\
& \left({ }^{-1} u_{g}^{-1} \cdot{ }^{-1} m \cdot{ }^{-1}{ }^{\prime} a_{g}^{\prime}\right),
\end{aligned}
$$

for all $a \in A^{\otimes n}, \sigma, \tau \in S_{n}, m \in M^{\otimes n}, a_{g}^{\prime} \in A_{g}^{\prime \otimes n}, g \in \bar{G}^{n}$ and $u_{g}$ is an invertible homogeneous element of $A_{g}^{\otimes n}$. We start by proving that $f$ is a morphism of $\left(A \prec S_{n}, A^{\prime}\right.$ ? $S_{n}$ )-bimodules:

$$
\begin{aligned}
& f\left((b \otimes \tau) \cdot\left((a \otimes \sigma) \otimes_{B \otimes n} m\right)\right)=f\left(((b \otimes \tau) \cdot(a \otimes \sigma)) \otimes_{B \otimes n} m\right) \\
& =f\left(\left(b \cdot{ }^{\tau} a \otimes \tau \sigma\right) \otimes_{B} \otimes n m\right) \\
& =b \cdot{ }^{\tau} a \cdot{ }^{\tau \sigma} m \otimes \tau \sigma \\
& =b \cdot{ }^{\tau}\left(a \cdot{ }^{\sigma} m\right) \otimes \tau \sigma \\
& =(b \otimes \tau)\left(a \cdot{ }^{\sigma} m \otimes \sigma\right) \\
& =(b \otimes \tau) f\left((a \otimes \sigma) \otimes_{B \otimes n} m\right), \\
& f\left(\left((a \otimes \sigma) \otimes_{B \otimes n} m\right) \cdot\left(a_{g}^{\prime} \otimes \tau\right)\right) \\
& =f\left(\left(a \cdot{ }^{\sigma} u_{g} \otimes \sigma \tau\right) \otimes_{B \otimes n}\left({ }^{\tau^{-1}} u_{g}^{-1} \cdot \tau^{-1} m \cdot{ }^{\tau^{-1}} a_{g}^{\prime}\right)\right) \\
& =\left(a \cdot{ }^{\sigma} u_{g} \cdot{ }^{\sigma \tau}\left({ }^{\tau^{-1}} u_{g}^{-1} \cdot \tau^{-1} m \cdot{ }^{-1} a_{g}^{\prime}\right)\right) \otimes \sigma \tau \\
& =\left(a \cdot{ }^{\sigma} u_{g} \cdot{ }^{\sigma \tau \tau^{-1}} u_{g}^{-1} \cdot{ }^{\sigma \tau \tau^{-1}} m \cdot{ }^{\sigma \tau \tau^{-1}} a_{g}^{\prime}\right) \otimes \sigma \tau \\
& =\left(a \cdot{ }^{\sigma} u_{g} \cdot{ }^{\sigma} u_{g}^{-1} \cdot{ }^{\sigma} m \cdot{ }^{\sigma} a_{g}^{\prime}\right) \otimes \sigma \tau \\
& =\left(a \cdot{ }^{\sigma} m \cdot{ }^{\sigma} a_{g}^{\prime}\right) \otimes \sigma \tau \\
& =\left(\left(a \cdot{ }^{\sigma} m\right) \otimes \sigma\right)\left(a_{g}^{\prime} \otimes \tau\right) \\
& =f\left((a \otimes \sigma) \otimes_{B} \otimes n m\right)\left(a_{g}^{\prime} \otimes \tau\right),
\end{aligned}
$$

for all $a, b \in A^{\otimes n}, \sigma, \tau \in S_{n}, m \in M^{\otimes n}, a_{g}^{\prime} \in A_{g}^{\prime \otimes n}, g \in \bar{G}^{n}$ and $u_{g}$ is an invertible homogeneous element of $A_{g}^{\otimes n}$.

Next, we will prove that $f$ is $\bar{G}\left\{S_{n}\right.$-grade preserving. We recall from [3, Proposition 2.11] that the $\bar{G} \succ S_{n}$-grading of $\left(A \succ S_{n}\right) \otimes_{B \otimes n} M^{\otimes n}$ is given by $A \succ S_{n}$. We have:

$$
\begin{aligned}
& f\left(\left(\left(a_{g_{1}} \otimes \ldots \otimes a_{g_{n}}\right) \otimes \sigma\right) \otimes \otimes_{B} \otimes n\right. \\
& \quad=\left(\left(a_{g_{1}} m_{\sigma^{-1}(1)} \otimes \ldots \otimes a_{g_{n}} m_{\sigma^{-1}(n)}\right) \otimes \sigma\right. \\
& \quad \in\left(\left(A_{g_{1}} \tilde{M}_{1} \otimes \ldots \otimes m_{n}\right)\right) \\
& \quad \subseteq\left(\left(\tilde{M}_{g_{1}} \otimes \ldots \otimes \tilde{M}_{g_{n}}\right) \otimes \mathcal{O} \sigma\right. \\
& \quad=\left(\tilde{M} 2 S_{n}\right)_{\left(\left(g_{1}, \ldots, g_{n}\right), \sigma\right)},
\end{aligned}
$$


for all $\left(a_{g_{1}} \otimes \ldots \otimes a_{g_{n}}\right) \otimes \sigma \in\left(A \curlywedge S_{n}\right)_{\left(\left(g_{1}, \ldots, g_{n}\right), \sigma\right)}, m_{1} \otimes \ldots \otimes m_{n} \in M^{\otimes n}$ and for all $\left(\left(g_{1}, \ldots, g_{n}\right), \sigma\right) \in \bar{G} \imath S_{n}$.

Finally, we will prove that $f$ is bijective. Because both modules have the same $\mathcal{O}$-rank, it is enough to prove that $f$ is surjective. If $\left(\tilde{m}_{g_{1}} \otimes \ldots \otimes \tilde{m}_{g_{n}}\right) \otimes \sigma$ is an arbitrary element of $\left(\tilde{M}<S_{n}\right)_{\left(\left(g_{1}, \ldots, g_{n}\right), \sigma\right)}$, then it is clear that

$\left(\left(u_{g_{1}^{-1}}^{-1} \otimes \ldots \otimes u_{g_{n}^{-1}}^{-1}\right) \otimes \sigma\right) \otimes\left(u_{g_{\sigma(1)}^{-1}} \tilde{m}_{g_{\sigma(1)}} \otimes \ldots \otimes u_{g_{\sigma(n)}^{-1}} \tilde{m}_{g_{\sigma(n)}}\right) \in\left(A 乙 S_{n}\right) \otimes_{B^{\otimes n}} M^{\otimes n}$

and that

$$
\begin{aligned}
& f\left(\left(\left(u_{g_{1}^{-1}}^{-1} \otimes \ldots \otimes u_{g_{n}^{-1}}^{-1}\right) \otimes \sigma\right) \otimes\left(u_{g_{\sigma(1)}^{-1}} \tilde{m}_{g_{\sigma(1)}} \otimes \ldots \otimes u_{g_{\sigma(n)}^{-1}} \tilde{m}_{g_{\sigma(n)}}\right)\right) \\
& =\left(\left(u_{g_{1}^{-1}}^{-1} \otimes \ldots \otimes u_{g_{n}^{-1}}^{-1}\right) \cdot{ }^{\sigma}\left(u_{g_{\sigma(1)}^{-1}} \tilde{m}_{g_{\sigma(1)}} \otimes \ldots \otimes u_{g_{\sigma(n)}^{-1}} \tilde{m}_{g_{\sigma(n)}}\right)\right) \otimes \sigma \\
& =\left(\left(u_{g_{1}^{-1}}^{-1} \otimes \ldots \otimes u_{g_{n}^{-1}}^{-1}\right) \cdot\left(u_{g_{1}^{-1}} \tilde{m}_{g_{1}} \otimes \ldots \otimes u_{g_{n}^{-1}} \tilde{m}_{g_{n}}\right)\right) \otimes \sigma \\
& =\left(u_{g_{1}^{-1}}^{-1} u_{g_{1}^{-1}} \tilde{m}_{g_{1}} \otimes \ldots \otimes u_{g_{n}^{-1}}^{-1} u_{g_{n}^{-1}} \tilde{m}_{g_{n}}\right) \otimes \sigma \\
& =\left(\tilde{m}_{g_{1}} \otimes \ldots \otimes \tilde{m}_{g_{n}}\right) \otimes \sigma,
\end{aligned}
$$

for all $\left(\left(g_{1}, \ldots, g_{n}\right), \sigma\right) \in \bar{G} 々 S_{n}$, where $u_{g}$ represents an invertible homogeneous element of $A_{g}$, for all $g \in \bar{G}$.

(3) Furthermore, by Proposition 3.3, $\tilde{M}^{\otimes n}$ is a $\bar{G}^{n}$-graded $\left(A^{\otimes n}, A^{\prime \otimes n}\right)$-bimodule over $\mathcal{C}^{\otimes n}$, which induces a $\bar{G}^{n}$-graded Morita equivalence over $\mathcal{C}^{\otimes n}$ between $A^{\otimes n}$ and $A^{\otimes n}$, thus by [1, Theorem 5.1.2] with respect to the $\bar{G}^{n}$-grading, we have that $\left(\tilde{M}^{\otimes n}\right)_{1}=M^{\otimes n}$ is a $\left(B^{\otimes n}, B^{\prime \otimes n}\right)$-bimodule, which induces a Morita equivalence between $B^{\otimes n}$ and $B^{\prime \otimes n}$.

Now, by the previous statements, and by using [3, Theorem 3.3] with respect to the $\bar{G}\left\{S_{n}\right.$-grading, we obtain that $\tilde{M} \curlyvee S_{n}$ induces a $\bar{G} \imath S_{n}$-graded Morita equivalence over $\mathcal{C}^{\otimes n}$ between $A$ ? $S_{n}$ and $A^{\prime} \supsetneq S_{n}$.

Acknowledgment. This work was supported by a grant of the Romanian Ministry of Research, Innovation and Digitalization, CNCS/CCCDI-UEFISCDI, project number PN-III-P1-1.1-TE-2019-0136, within PNCDI III.

\section{References}

[1] Marcus, A., Representation Theory of Group-Graded Algebras, Nova Science Publ. Inc., 1999.

[2] Marcus, A., Minuţă, V.A., Group graded endomorphism algebras and Morita equivalences, Mathematica, 62(85)(2020), no. 1, 73-80.

[3] Marcus, A., Minuţă, V.A., Character triples and equivalences over a group graded Galgebra, J. Algebra, 565(2021), 98-127.

[4] Minuţă, V.A., Graded Morita theory over a G-graded G-acted algebra, Acta Univ. Sapientiae Math., 12(2020), no. 1, 164-178.

[5] Späth, B., A reduction theorem for Dade's projective conjecture, J. Eur. Math. Soc. (JEMS), 19(2017), no. 4, 1071-1126.

[6] Späth, B., Inductive Conditions for Counting Conjectures via Character Triples, in Representation Theory - Current Trends and Perspectives, (H. Krause, P. Littelmann, G. Malle, K.H. Neeb, C. Schweigert, Eds.), EMS Ser. Congr. Rep., Zürich, 2017, 665-680. 
[7] Späth, B., Reduction theorems for some global-local conjectures, in Local Representation Theory and Simple Groups, (R. Kessar, G. Malle, D. Testerman, Eds.), EMS Ser. Lect. Math., Zürich, 2018, 23-61.

Virgilius-Aurelian Minuţă

Technical University of Cluj-Napoca,

Faculty of Automation and Computer Science,

Department of Mathematics,

25, G. Bariţiu Street, 400027,

Cluj-Napoca, Romania

Babeş-Bolyai University,

Faculty of Mathematics and Computer Science,

Department of Mathematics,

1, M. Kogălniceanu Street, 400084,

Cluj-Napoca, Romania

e-mail: minuta.aurelian@math.ubbcluj.ro 